\title{
Wnt7a-releasing synthetic hydrogel enhances local skeletal muscle regeneration and muscle stem cell engraftment
}

Woojin M. Han ${ }^{1,2}$, Mahir Mohiuddin ${ }^{1,3}$, Shannon E. Anderson ${ }^{1,3}$, Andrés J. García ${ }^{\ddagger, 1,2}$, and Young C. Jang ${ }^{\ddagger 1,3,4}$

1. Parker H. Petit Institute for Bioengineering and Bioscience, Georgia Institute of Technology, Atlanta, GA

2. The George W. Woodruff School of Mechanical Engineering, Georgia Institute of Technology, Atlanta, GA

3. The Wallace H. Coulter Department of Biomedical Engineering, Georgia Institute of Technology, Atlanta, GA

4. School of Biological Sciences, Georgia Institute of Technology, Atlanta, GA

‡Co-corresponding authors:

Andrés J. García, Ph.D.

Executive Director, Parker H. Petit Institute for Bioengineering and Bioscience

Petit Director's Chair in Bioengineering and Bioscience

Regents' Professor, Woodruff School of Mechanical Engineering

Petit Institute for Bioengineering and Bioscience

Georgia Institute of Technology

315 Ferst Drive NW

Atlanta, GA 30332-0363

E-mail: andres.garcia@me.gatech.edu

\section{Young C. Jang, Ph.D.}

Assistant Professor, School of Biological Sciences

Petit Institute for Bioengineering and Bioscience

Georgia Institute of Technology

315 Ferst Drive, 3304 Petit Biotechnology Building

Atlanta, GA 30332-0363

E-mail: young.jang@gatech.edu 


\section{ABSTRACT}

Skeletal muscle possesses efficient ability to regenerate upon minor injuries, but its capacity to regenerate is severely compromised with traumatic injuries and muscle-associated diseases. Recent evidence suggests that skeletal muscle regeneration can be accelerated by transplantation of muscle satellite cells (MuSCs) or treatment with promyogenic factors, such as Wingless-type MMTV Integrated 7a (Wnt7a) protein. Although direct intramuscular injection is the simplest method to deliver MuSCs and Wnt7a for regenerative therapy, direct injection is not viable in many clinical cases where the structural integrity is severely compromised. To address this challenge, we engineered a synthetic poly(ethylene glycol) (PEG)-based hydrogel to facilitate the co-delivery of pro-myogenic factors, such as Wnt7a, and MuSCs to skeletal muscles affected by severe trauma or muscular dystrophies. Wnt7a release rate can be controlled by modulating the polymer density of the hydrogel, and this release rate can be further accelerated through the proteolytic degradation of the hydrogel. Treating cryo-injured tibialis anterior (TA) muscles with Wnt7a-loaded hydrogels resulted in an accelerated regenerative response, measured by increased muscle fiber cross-sectional area, bulk TA mass, and number of $\mathrm{Pax}^{+}$MuSCs at the injury site, compared to the TA muscles treated with Wnt7a-free hydrogels. Co-delivery of Wnt7a and primary MuSCs using the synthetic hydrogel to the cryo-injured TA muscles significantly increased cellular migration during the engraftment process. This work provides a synthetic biomaterial platform for advancing treatment strategies of skeletal muscle trauma and diseases, specifically in conditions where direct intramuscular injection may be challenging. 
bioRxiv preprint doi: https://doi.org/10.1101/569921: this version posted March 6, 2019. The copyright holder for this preprint (which was not

certified by peer review) is the author/funder, who has granted bioRxiv a license to display the preprint in perpetuity. It is made available under aCC-BY-NC-ND 4.0 International license.

\section{INTRODUCTION}

Skeletal muscle plays an indispensable role in locomotion and metabolism. Skeletal muscle regenerates efficiently following minor injuries, but its repair capacity is severely challenged with the onset of acute muscle trauma (e.g., laceration and volumetric muscle loss) and chronic muscle diseases (e.g., Duchenne muscular dystrophy) $(1,2)$. Inadequate muscle regeneration leads to incapacitating pathophysiologic consequences, such as aberrant inflammation, excessive fibrosis, and scar formation, ultimately resulting in permanent loss of muscle function, disability, and diminished quality of life $(3,4)$. Current muscle reconstruction strategies include surgical suturing and autologous muscle graft, but these approaches do not completely repair the injured muscle $(3,5)$. In addition, autologous muscle transplant causes donor site morbidity and is prone to graft failure, especially in aged patients with muscle wasting conditions (3). Effective therapeutic strategies for promoting skeletal muscle regeneration remain to be developed.

Recent studies have revealed that Wingless-type MMTV Integrated 7a (Wnt7a) protein, a ligand for Frizzled (Fzd) receptors, functions as a potent mediator of muscle growth and repair (6-10). Notably, Wnt7a induces myofiber hypertrophy through the Akt/mTOR protein synthesis pathway $(8,9)$, promotes symmetric expansion $(6,10)$, and migration of muscle satellite cells (MuSCs) through the planar cell polarity pathway involving Dishevelled 2 (Dvl2) and the small GTPase Rac1 $(6,7,10)$. Furthermore, pre-treatment of MuSCs with Wnt7a significantly enhances MuSC dispersion and engraftment upon cellular transplantation (7). In a rodent model of Duchenne muscular dystrophy (DMD), administration of recombinant human Wnt7a into tibialis anterior muscles significantly promotes MuSC expansion, myofiber hypertrophy, and muscle strength, suggesting that Wnt7a serves as an effective pro-myogenic factor for stimulating muscle repair (9). Although Wnt7a treatment may also enhance local muscle regeneration upon injury, traumatic injuries are often characterized by a severe loss in tissue integrity $(11,12)$, rendering direct injection of Wnt7a and other therapeutics not feasible. Therefore, there is a need for a delivery vehicle that enables controlled administration of therapeutics to the injured or pathologic muscles where direct injection may not be applicable.

Hydrogel-based biomaterials offer a promising strategy to deliver therapeutics, including stem/progenitor cells and growth factors (13-15). These biomaterial systems enable precise control over the spatiotemporal release of therapeutic molecules and address clinical challenges associated with requiring high dosages, repeated administration, and short half-lives of protein-based agents (13). Recently, we engineered a synthetic bioactive hydrogel based on a four-arm poly(ethylene glycol) (PEG) macromer with its ends functionalized with maleimide groups (PEG-4MAL) for delivering MuSCs to dystrophic and aged skeletal muscles with comorbid trauma (16). The engineered PEG-4MAL hydrogel provides several advantages for treating muscle trauma and disorders, including its fully synthetic nature that increases its translational potential, and ability to systematically control the structure, physical and biochemical properties, and degradability (16-18). Furthermore, the engineered PEG-4MAL hydrogel efficiently adheres to biological tissue through maleimide-thiol interactions (Michael-type addition) at physiological conditions (18), making the system suitable for locally delivering cells and therapeutics to the injured or pathologic muscles where direct injection is impractical. We also demonstrated that the engineered PEG-4MAL hydrogel supports primary MuSC function, and MuSC delivery using the material significantly improves their engraftment efficiency compared to hydrogel-free and collagen 
gel controls (16). However, the effect of co-delivering pro-myogenic growth factors, such as Wnt7a, with MuSC using the engineered PEG-4MAL hydrogel on stimulating muscle regeneration has not yet been evaluated.

In this study, we engineered a synthetic PEG-4MAL hydrogel to facilitate the co-delivery of Wnt7a and MuSCs for accelerated skeletal muscle healing. We demonstrate that the release rate of Wnt7a can be controlled by modulating the hydrogel polymer density, and the hydrogel-released Wnt7a retains its bioactivity both in vitro and in vivo.

Supramuscular delivery of Wnt7a-releasing hydrogel to the freeze-injured tibialis anterior (TA) muscle accelerates the regenerative response by promoting muscle fiber hypertrophy and increasing endogenous Pax $7^{+}$MuSCs at the site of injury. Finally, we demonstrate that the co-delivery of Wnt7a and MuSCs using the engineered PEG-4MAL hydrogel synergistically enhances the migration capacity of donor MuSCs into the injured TA muscle. Collectively, we establish codelivery of Wnt7a and MuSCs using the engineered synthetic PEG-4MAL hydrogel as a potential therapeutic strategy to locally treat skeletal muscle injuries and disorders where direct injection may not be applicable. 


\section{MATERIALS AND METHODS}

\section{Animals}

All live animal procedures were conducted under the approval of the Institutional Animal Care and Use Committee of the Georgia Institute of Technology. Mice were housed, maintained, and bred in the Physiological Research Laboratory Animal Facility of the Georgia Institute of Technology. C57BI/6 and $m d x$ (B6Ros.Cg-DMD $\left.{ }^{m d x}-4 C V / J\right)$ mice were obtained from the Jackson Laboratory. CAG-luc-Ka- $\beta$-actin-EGFP mice were generated by crossing C57BI/6- $\beta$-actin-EGFP (kindly provided by Amy Wagers, Harvard University) and FVB-Tg(CAG-luc,-GFP)L2G85Chco/J (acquired from the Jackson Laboratory) mice. These mice were back-crossed for more than 5 generations and maintained in C57BI/6 background. Both male and female mice were used in a randomized manner.

\section{Synthesis of Wnt7a-loaded hydrogel}

Four-arm PEG-4MAL macromer (20 kDa, Laysan Bio) was reconstituted in 1X phosphate-buffered saline (PBS) containing 10 mM HEPES (pH 7.4). Cell adhesive peptides (GRGDSPC, or scrambled GRDGSPC; >95\% purity; GenScript) were dissolved in PBS containing 10 mM HEPES. To generate peptide-functionalized PEG-4MAL precursors, cell adhesive peptides were mixed with the PEG-4MAL solution. Subsequently, recombinant human Wnt7a (R\&D systems) reconstituted in PBS was added to the PEG-4MAL precursor solution. To synthesize Wnt7a-loaded hydrogel, proteasedegradable cross-linking peptide (GCRDVPMSMRGGDRCG; >95\% purity; GenScript) dissolved in PBS containing 10 mM HEPES was mixed with the PEG-4MAL precursor solution containing Wnt7a and polymerized at $37^{\circ} \mathrm{C}$ for 5 min.

\section{Release assay}

Wnt7a was labeled with AlexaFluor 488 NHS ester (ThermoFisher Scientific) per the manufacturer's instructions. 1 $\mu \mathrm{g}$ labeled-Wnt7a was encapsulated in a $15 \mu \mathrm{L}$ PEG-4MAL hydrogel and subsequently submerged in $1 \mathrm{~mL}$ PBS. Release kinetics of Wnt7a from hydrogels was fluorescently quantified over time at $37^{\circ} \mathrm{C}$ by sampling the bathing solution in a 24-well microvolume microplate and SpectraMax M3 reader (Molecular Devices). For each time point, PBS (blank control) and free $1 \mu \mathrm{g}$ labeled-Wnt7a in $1 \mathrm{~mL}$ PBS (100\% control) were also assayed. To degrade Wnt7a-loaded hydrogels, the hydrogels were incubated in collagenase I (3.9 U/mL in PBS; Worthington Biochemical Corporation) at $37^{\circ} \mathrm{C}$.

\section{In vitro bioactivity assay}

C2C12 myoblasts were cultured in $1.5 \mathrm{~mL}$ growth media (Dulbecco's modified Eagle's medium, DMEM containing $100 \mathrm{U} / \mathrm{mL}$ penicillin/streptomycin and $10 \%$ fetal bovine serum [FBS]) in 12-well plates until confluency. Subsequently, the growth media was replaced with $1.5 \mathrm{~mL}$ of differentiation media (DMEM containing $100 \mathrm{U} / \mathrm{mL}$ penicillin/streptomycin and 2\% horse serum) containing Wnt7a (75 ng)-loaded PEG-4MAL hydrogels (4\%, 6\%, 8\% w/v), free Wnt7a (75 ng), or PBS control. After 5 days of differentiation, myotubes were fixed using $4 \%$ paraformaldehyde for $20 \mathrm{~min}$ at room temperature, washed, treated with blocking/permeabilizing buffer (2\% bovine serum albumin [BSA], 
bioRxiv preprint doi: https://doi.org/10.1101/569921; this version posted March 6, 2019. The copyright holder for this preprint (which was not certified by peer review) is the author/funder, who has granted bioRxiv a license to display the preprint in perpetuity. It is made available under aCC-BY-NC-ND 4.0 International license.

0.5\% goat serum, and 0.5\% Triton X-100 in PBS), and stained for myosin heavy chain (1:200; MF20, Developmental Studies Hybridoma Bank) overnight at $4{ }^{\circ} \mathrm{C}$. The myotubes were washed 3 times using PBS containing Tween-20 $(0.1 \%$ $\mathrm{v} / \mathrm{v}$ ) and incubated with AlexaFluor 488-conjugated goat anti-mouse IgG (1:250; ThermoFisher) for 1 hour at room temperature. Samples were washed 3 times using PBS containing Tween-20 (0.1\% v/v) and imaged using an epifluorescent microscope (Zeiss Observer D1). To assess the degree of hypertrophy, myotube diameters were measured using Fiji ImageJ 1.52e (19).

\section{PEG-4MAL macromer muscle adhesion assay}

Scrambled RDG peptides (GRDGSPC; >95\% purity; GenScript) were labeled with DyLight 755 NHS ester (ThermoFisher Scientific) per the manufacturer's instructions. DyLight 755-labeled RDG-functionalized PEG-4MAL precursors (remaining maleimide concentration $=6.27 \times 10^{-3} \mathrm{~mol} / \mathrm{mL}$ ) were produced as described above. To generate maleimide-quenched precursors, excess L-cysteine (Millipore Sigma) dissolved in PBS was added to the PEG-4MAL solution. $15 \mu \mathrm{L}$ of PBS, macromer solution with quenched maleimides, or macromer solution with reactive maleimides was pipetted onto the TA surfaces of freshly sacrificed $\mathrm{mdx}-4 \mathrm{CV}$ mice. Treated TA muscles were harvested and washed for 5 min twice in PBS. Fluorescence measurement of the TAs was acquired using the IVIS SpectrumCT in vivo imaging system (PerkinElmer).

\section{MuSC isolation}

Primary MuSCs were isolated as described previously (16). Briefly, hindlimb muscles from 2-4-month-old mice were minced and digested in DMEM containing collagenase type II (0.2\%; Worthington Biochemical Corporation) and dispase II (2.5 U/mL; ThermoFisher) for $90 \mathrm{~min}$ at $37^{\circ} \mathrm{C}$ on a shaker. The muscle digest was triturated every 30 min using an FBScoated serological pipette. Upon digestion, the muscle digest was diluted by adding a two-part volume of $20 \%$ FBS in F10 media. The resulting solution was filtered through a $70 \mu \mathrm{m}$ cell strainer. Cell pellets were obtained by centrifuging ( $300 \mathrm{~g})$ the solution for $5 \mathrm{~min}$ at $4{ }^{\circ} \mathrm{C}$. The cell pellets were retrieved by removing supernatant and further washed using $2 \%$ FBS in Hank's balanced salt solution (HBSS) through 5 min centrifugation $(300 \mathrm{~g})$ at $4{ }^{\circ} \mathrm{C}$. To stain the cells with primary antibodies, a cocktail of primary antibodies (1:100) was added to the cell suspension solution in 2\% FBS in HBSS and incubated for $30 \mathrm{~min}$ on ice. The primary antibody cocktail consists of phycoerythrin (PE)-conjugated anti-mouse CD29 (13.3\%; BioLegend), allophycocyanin (APC)-conjugated anti-mouse Sca1 (13.3\%; BioLegend), CD45 (13.3\%; BioLegend), CD11b (13.3\%; BioLegend), CD31 (13.3\%; BioLegend), Ter119 (13.3\%; BioLegend), and biotinylated anti-mouse CD184 (20.2\%; BD Pharmingen). The cells were washed in 2\% FBS in HBSS through 5 min centrifugation $(300 \mathrm{~g})$ at $4{ }^{\circ} \mathrm{C}$. The cells were further incubated with streptavidin PE-Cy7 (1:100; eBioscience) for $20 \mathrm{~min}$ on ice and washed as described above. Immediately before cell sorting, propidium iodide (1:1000; ThermoFisher) was added to the cell solution. Propidium iodide $\mathrm{CD}^{-} \mathrm{C}^{+}, \mathrm{CD}_{184^{+}}, \mathrm{Sca} 1^{-}, \mathrm{CD} 31^{-}, \mathrm{CD} 45^{-}, \mathrm{CD} 11 \mathrm{~b}^{-}$, Ter119- MuSCs were sorted via fluorescence-activated cell sorting (BD FACSAria III Cell Sorter). 
bioRxiv preprint doi: https://doi.org/10.1101/569921; this version posted March 6 2019. The copyright holder for this preprint (which was not

certified by peer review) is the author/funder, who has granted bioRxiv a license to display the preprint in perpetuity. It is made available under aCC-BY-NC-ND 4.0 International license.

\section{D MuSC culture, proliferation, and migration assay}

Freshly isolated MuSCs were encapsulated in 4\% 20 kDa PEG-4MAL hydrogels (1.0 mM RGD, $50 \mathrm{ng} / \mathrm{mL}$ Wnt7a, VPM crosslinked) as described above. MuSCs were cultured in growth media (F10 containing $100 \mathrm{U} / \mathrm{mL}$ penicillin/streptomycin, $1 X$ GlutaMAX, and 20\% horse serum). For EdU staining, cells were incubated in $10 \mu \mathrm{M}$ EdUcontaining growth media for 5 hours. Subsequently, the cells were fixed in $4 \%$ paraformaldehyde for 20 min at room temperature and washed three times using PBS. The cells were blocked and permeabilized using PBS containing $2 \%$ BSA, $0.5 \%$ goat serum, and $0.5 \%$ Triton X-100 for 1 hour at room temperature. EdU detection was conducted per the manufacturer's instructions (ThermoFisher). Cell nuclei were stained with Hoechst (1:1000) prior to z-stack imaging using a confocal microscope (Nikon Eclipse Ti-E C2+). The number of nuclei and EdU ${ }^{+}$nuclei were automatically counted using CellProfiler (20). Total colony area per volume was quantified using ImageJ. To assess MuSC migration, 4\% 20 kDa PEG-4MAL hydrogels (1.0 mM RGD, VPM crosslinked) containing Wnt7a (50 ng) or PBS were cast in angiogenesis plate wells (ibidi), and freshly isolated 10,000 GFP+ MuSCs were seeded on top of the hydrogels. 1,360 $\mu \mathrm{m}$-thick z-stack projections were taken, from the top of the hydrogel, using a confocal microscope (Nikon Eclipse Ti-E C2+). Cell migration distance was quantified using ImageJ.

\section{Freeze injury and Wnt7a/MuSC delivery}

Mice were anesthetized by inhalation of $2.5 \%$ isoflurane. Sustained-release buprenorphine $(0.8 \mathrm{mg} / \mathrm{kg})$ was subcutaneously administered immediately after induction. The hindlimb hair was depilated to expose the skin. To expose the TA muscle, an incision was made through the skin and fascia between the TA and gastrocnemius muscles on the lateral side. A liquid nitrogen-cooled metal probe was positioned on the TA surface for 10 sec to induce freeze injury. Upon muscle recovery, $15 \mu \mathrm{L}$ PEG-4MAL hydrogel (4\% 20 kDa PEG-4MAL, 1.0 mM RGD, VPM crosslinked) containing 2.5 $\mu \mathrm{g}$ Wnt7a or PBS was cast on the injured TA of $\mathrm{C} 57 \mathrm{BI} / 6$ mice. For hydrogel-mediated co-delivery of Wnt7a and freshly isolated GFP ${ }^{+}$MuSCs, 15 LL PEG-4MAL hydrogel (3\% 20 kDa PEG-4MAL, 1.0 mM RGD, VPM crosslinked) containing 300 ng Wnt7a with MuSCs or PBS with MuSCs was cast on the injured TA of $m d x$ mice. The skin incision was closed using degradable surgical suture (Vicryl suture, Ethicon). All procedures were conducted using aseptic techniques.

\section{Tissue histology and immunostaining}

Hindlimbs were fixed in $4 \%$ paraformaldehyde for 1 hour at room temperature. Fixed hindlimbs were washed in PBS three times and incubated in $20 \%$ sucrose (w/v in PBS) overnight at $4{ }^{\circ} \mathrm{C}$. Dissected TA muscles were frozen in liquid nitrogen-cooled 2-methylbutane. $10 \mu$ m-thick tissue sections were acquired through cryo-sectioning. Prior to tissue staining, the sectioned tissues were treated with blocking/permeabilizing buffer ( $2 \%$ BSA, $0.5 \%$ goat serum, and $0.5 \%$ Triton X-100 in PBS) for 1 hour at room temperature.

To stain for Pax7, TA muscles were frozen unfixed. $10 \mu \mathrm{m}$-thick tissue sections were obtained and fixed using 4\% paraformaldehyde for $8 \mathrm{~min}$. Heat activated antigen retrieval was performed by incubating the tissues in citrate buffer (pH 6.0) and treating in a high-pressure cooker for $10 \mathrm{~min}$. The tissue sections were washed using PBS, blocked using 
blocking/permeabilizing buffer (2\% BSA, $0.5 \%$ goat serum, and $0.5 \%$ Triton $\mathrm{X}-100)$ for 1 hour at room temperature, and finally incubated in blocking/permeabilizing buffer containing AffiniPure Fab goat anti-mouse-lgG (1:25; Jackson ImmunoResearch Laboratories, Inc.) for 1-2 hours at room temperature

The following primary and secondary antibodies were used to stain tissue sections in this study: rabbit polyclonal anti-dystrophin (1:200; Abcam), Pax7 (1:80, Developmental Studies Hybridoma Bank), Alexa Fluor 488-conjugated antiGFP (1:250; ThermoFisher), Alexa Fluor 555 goat anti-mouse IgG (1:250; ThermoFisher), Alexa Fluor 488 goat anti-rabbit IgG (1:250; ThermoFisher). Hoechst 33342 (1:1000; Thermo Fisher) was used to counterstain cell nuclei.

\section{Pax $7^{+}$MuSC and myofiber cross-sectional area quantification}

To quantify Pax $7^{+}$MuSCs, tissue sections were imaged and manually quantified in a blinded manner, where the slide labels were physically masked using a nontransparent tape by a different researcher who did not partake in the imaging and quantification process. The number of $\mathrm{Pax} 7^{+}$MuSCs were scored from 8 images per muscle. Myofiber crosssectional area was automatically quantified using ImageJ. Briefly, the dystrophin images were converted 8-bit and automatic threshold was applied. Myofiber cross-sectional areas were then automatically quantified using the Analyze Particle function.

\section{Statistical analyses}

Statistical tests were conducted using GraphPad Prism 8. Normality was tested using the Shapiro-Wilk test. Two-way repeated measures ANOVA with Tukey's multiple comparisons tests were performed on the Wnt7a release data. Twoway ANOVA with Sidak's multiple comparisons tests was performed to myogenic colony formation and EdU incorporation assay data. For all other multiple group comparisons, one-way ANOVA with Tukey's tests or Kruskal-Wallis test with Dunn's tests were performed based on data normality. For the paired comparisons, two-tailed paired t-tests were conducted. Two-tailed unpaired t-tests were performed for all other two-group comparisons. Significance levels were set at ${ }^{*} p<0.05, * * p<0.01, * * * p<0.01, * * * * p<0.001$. 
bioRxiv preprint doi: https://doi.org/10.1101/569921: this version posted March 6 2019. The copyright holder for this preprint (which was not certified by peer review) is the author/funder, who has granted bioRxiv a license to display the preprint in perpetuity. It is made available under aCC-BY-NC-ND 4.0 International license.

\section{RESULTS}

\section{Synthetic PEG-4MAL hydrogel for controlled-release of Wnt7a}

To engineer a Wnt7a-releasing hydrogel, we encapsulated Wnt7a in a hydrogel composed of a four-arm poly(ethylene glycol) (PEG) macromer with its ends functionalized with maleimide groups (PEG-4MAL). Previously, we demonstrated that PEG-4MAL hydrogels can be customized to effectively deliver growth factors for various tissue regeneration applications, including vascular endothelial growth factor for pancreatic islet vascularization (21-23) and bone morphogenetic protein-2 for bone regeneration (24). Furthermore, PEG-4MAL hydrogels exhibit excellent biocompatibility in vivo $(16,18,23,25)$. In skeletal muscles, the engineered PEG-4MAL platform supports primary MuSC function and serves as an effective delivery vehicle for MuSC transplantation (16). In this system, the maleimide functional groups enable stoichiometric incorporation of thiol-presenting cell-adhesive ligands through Michael-type addition (Fig. 1a). Functionalized macromers can then be polymerized using cysteine-flanked protease-degradable peptides, which results in a hydrogel with a highly defined network structure that exhibits "on-demand" degradability (Fig. 1a). Furthermore, hydrogel mesh size, which governs solute diffusion through the hydrogel network, can be decreased by increasing the polymer density, permitting precise control of growth factor release rate.

Wnt7a release rate can be modulated by encapsulating in PEG-4MAL hydrogels with different polymer densities. The release rate of Wnt7a through passive diffusion is significantly delayed in $8 \%(w / v)$ PEG-MAL hydrogel compared to 6\% PEG-4MAL hydrogel (Fig. 1b; $p<0.0001$ ) in the absence of collagenase; this result is expected based on the tighter network mesh for the higher polymer density gels. The release rate of Wnt7a is further increased in presence of protease (i.e., $3.9 \mathrm{U} / \mathrm{mL}$ of collagenase type I; Fig. $1 \mathrm{~b} ; p<0.0001$ ), indicating that the release rate can be further enhanced through proteolytic degradation of the hydrogel.

\section{Hydrogel-released Wnt7a retains its bioactivity in vitro}

To determine whether hydrogel-released Wnt7a retains its bioactivity, we assessed the capacity of hydrogelreleased Wnt7a to promote in vitro myotube hypertrophy (Fig. 2a). In differentiated myotubes, Wnt7a binds to Fzd7 and activates the Akt/mTOR protein synthesis pathway to induce myotube hypertrophy (8). Significant hypertrophy of C2C12 myotubes was observed with treatment with free Wnt7a (Fig. 2b, c, g; $p<0.05$ ) compared to PBS control. Treating C2C12 myotubes with Wnt7a-releasing 4\% PEG-4MAL hydrogel resulted in a comparable degree of hypertrophy as free Wnt7a (Fig. 2c, d, g; $p>0.05$ ). Treatment with Wnt7a-loaded 6\% and 8\% PEG-4MAL hydrogels resulted in decreased levels of myotube hypertrophy, compared to both free Wnt7a and 4\% PEG-4MAL conditions (Fig. 2c, d, e, f, g; $p<0.05$ ). The distribution of myotube diameter increased with decreasing hydrogel polymer density (Fig. 2h), further suggesting that the effect of delivered Wnt7a can be temporally modulated by altering the Wnt7a release kinetics (Fig. 1b). Collectively, these data indicate that hydrogel-released Wnt7a retains its bioactivity in vitro, and further suggest that the release rate of Wnt7a modulates myotube hypertrophy.

\section{Engineered PEG-4MAL hydrogel enables effective local delivery of Wnt7a to the injured skeletal muscle}


bioRxiv preprint doi: https://doi.org/10.1101/569921; this version posted March 6, 2019. The copyright holder for this preprint (which was not

certified by peer review) is the author/funder, who has granted bioRxiv a license to display the preprint in perpetuity. It is made available under aCC-BY-NC-ND 4.0 International license.

The PEG-4MAL hydrogel can adhere to the tissue through maleimide-thiol interactions at physiologic conditions, and thus offers a unique advantage for locally administering therapeutics at the site of tissue injury where direct injection is not practical (Fig. 3a). Indeed, retention of unpolymerized, reacting PEG-4MAL macromers on the surface of TA muscle is significantly higher compared to unpolymerized macromers with quenched maleimides $(p<0.05)$ and untreated $(p<0.01$; Fig. $3 \mathrm{~b}$ and c), corroborating the feasibility of adhering a Wnt7a-loaded PEG-4MAL hydrogel to skeletal muscle.

To further assess the feasibility of promoting muscle regeneration by locally administering Wnt7a in severe muscle injury, we delivered Wnt7a-loaded hydrogels to the supramuscular locus of freeze-injured TA muscle. The muscles treated with Wnt7a-loaded hydrogels exhibited significantly increased median myofiber area at the site of injury compared to the muscles treated with Wnt7a-free hydrogels (Fig. 4a and b; $p<0.05$ ). Treatment with Wnt7a-loaded hydrogels also significantly increased TA mass compared to TAs treated with Wnt7a-free control hydrogels (Fig. 4c; $p<0.01)$. Furthermore, a significantly higher number of endogenous Pax7 ${ }^{+}$MuSCs were found in the TAs treated with Wnt7a-loaded hydrogels compared to control gels (Fig. $4 \mathrm{~d}$ and e; $p<0.05$ ). Collectively, these results suggest that hydrogel-release Wnt7a retains its bioactivity in vivo, and that local delivery of Wnt7a to severely injured muscle using the engineering PEG-4MAL hydrogel is feasible and effective in accelerating muscle regeneration.

\section{Wnt7a promotes MuSC migration through the engineered PEG-4MAL hydrogel}

Wnt7a promotes symmetric expansion and migration of MuSCs through the planar cell polarity pathway involving Dvl2 and the small GTPase Rac1 $(6,7,10)$. Furthermore, brief treatment of MuSCs prior to transplantation enhances engraftment (7), supporting the application of hydrogel-mediated MuSCs transplantation with Wnt7a to treat muscle trauma. We have previously engineered a PEG-4MAL hydrogel that is conducive to primary MuSC function and demonstrated that MuSC transplantation using the engineered hydrogel significantly improves the engraftment potential (16). To determine how Wnt7a influences MuSC proliferation within the engineered hydrogel in vitro, we encapsulated freshly isolated MuSCs in hydrogels presenting either RGD or RDG (scrambled inactive control) integrintargeting ligands with or without Wnt7a. Myogenic colonies formed in the RGD-presenting hydrogel were significantly larger than the colonies formed in the RDG-presenting hydrogels (Fig. 5a and b; $p<0.001$ ). However, encapsulated Wnt7a did not result in an increased size of myogenic colony formation in both RGD- and RDG-functionalized hydrogels (Fig. 5a and b). Although no significant difference in EdU incorporation was observed within the RGD-presenting gels with encapsulated Wnt7a, the number of EdU ${ }^{+}$nuclei per colony in the Wnt7a-treated RGD-presenting hydrogel was significantly higher than both RDG-functionalized conditions (Fig. 5a and c; $p<0.01$ ). Furthermore, no difference in colony density was observed in RGD-presenting hydrogels with encapsulated Wnt7a compared to control RGD-presenting gels (Fig. 5d and e), indicating that Wnt7a does not increase the proliferation capacity of myogenic progenitors in the engineered PEG-4MAL hydrogel. However, MuSCs seeded on top of Wnt7a-releasing hydrogel exhibited increased invasion distance into the hydrogel compared to MuSCs seeded on Wnt7a-free hydrogel (Fig. $5 f$ and g). This result shows that Wnt7a promotes MuSCs migration through the engineered synthetic matrix, which could further improve transplantation efficacy when delivered to the injured muscle in vivo. 


\section{Wnt7a promotes MuSC migration in vivo}

Delivery of MuSCs using the engineered PEG-4MAL hydrogel to the supramuscular locus of freeze-injured TA muscle $(m d x)$ significantly increases engraftment potential compared to the hydrogel-free condition (Fig. 6a and b), consistent with our previous findings (16). To further evaluate the effect of Wnt7a on MuSC engraftment, we next delivered freshly isolated GFP+ MuSCs using the engineered PEG-4MAL hydrogels with or without encapsulated Wnt7a to the supramuscular locus of freeze-injured TA muscles. Transplantation of MuSCs via the engineered PEG-4MAL hydrogel containing Wnt7a did not increase the number of GFP ${ }^{+}$fibers engrafted (Fig. $6 c$ and $d ; p>0.05$ ). However, the minimal distance of $\mathrm{GFP}^{+}$engrafted fibers from the TA surface was significantly increased when MuSCs were co-delivered with Wnt7a via the PEG-4MAL hydrogel (Fig. 6c and e), suggesting that MuSCs delivered in the absence of Wnt7a preferentially engraft along the supramuscular locus, while MuSCs co-delivered with Wnt7a engraft at a deeper location from the TA surface and the delivery site. Taken together, the data demonstrate that co-delivery of MuSCs and Wnt7a to the supramuscular locus of injured TA muscle using the engineered PEG-4MAL hydrogel promotes MuSC in vivo migration without impacting the engraftment efficiency. 
bioRxiv preprint doi: https://doi.org/10.1101/569921; this version posted March 6, 2019. The copyright holder for this preprint (which was not

certified by peer review) is the author/funder, who has granted bioRxiv a license to display the preprint in perpetuity. It is made available under aCC-BY-NC-ND 4.0 International license.

\section{DISCUSSION}

MuSC transplantation and pro-myogenic factor delivery are effective therapeutic strategies to regenerate damaged and diseased skeletal muscles, but direct delivery of cells and proteins to severely injured (e.g., laceration and volumetric muscle loss) muscles is challenging due to a loss in tissue integrity. To address this challenge, we engineered a synthetic PEG-4MAL hydrogel to facilitate the co-delivery of MuSCs and Wnt7a protein in conditions where direct injection may not be feasible. The maleimide groups functionalized on the PEG macromer efficiently react with thiolpresenting biological tissues to form stable covalent bonds via Michael-type addition, enabling hydrogel adhesion to the site of tissue injury and subsequently permit local delivery of cells and therapeutics (Fig. 3). We have previously demonstrated that the engineered PEG-4MAL hydrogel supports primary MuSC survival, proliferation, and differentiation, and enables delivery of MuSCs in dystrophic and aged skeletal muscles with comorbid trauma (16). The engineered PEG-4MAL hydrogel significantly improves the cellular engraftment efficiency compared to hydrogel-free and collagen gel controls (16). In this study, we further demonstrate that the engineered PEG-4MAL hydrogel enables controlled delivery of Wnt7a to accelerate the regenerative response by targeting the endogenous MuSCs and myofibers (Fig. 4). In addition, we establish the feasibility of co-delivering exogenous MuSCs and Wnt7a using the engineered PEG4MAL hydrogel (Fig. 6). Co-delivery of MuSCs and Wnt7a significantly increases the migration capacity of the donor MuSCs in vivo (Fig. 6), suggesting that the function of hydrogel-released MuSCs can be modulated by incorporation of pro-myogenic proteins and growth factors.

Hydrogel-mediated delivery of cells and protein-based therapeutics offer numerous advantages over vehicle-free cell and protein delivery methods $(13,15)$. In protein and drug delivery, hydrogels enable spatiotemporally controlled release of therapeutics and minimize the need for the repeated administration of high dosages. Hydrogel-based delivery also permits localized administration of therapeutics to the target tissue of interest, reducing the risk of systemic offtarget effects. In the current study, we show that the release rate of Wnt7a from the engineered PEG-4MAL hydrogel can be tuned by modulating the macromer density and protease-mediated degradation of the hydrogel (Fig. 1a). Delivery of Wnt7a-loaded PEG-4MAL hydrogel to the injured muscle significantly accelerated the regenerative response at the injury site by day 14 (Fig. 4). Although beyond the scope of the current study, different muscle types (e.g., craniofacial, diaphragm), injuries (e.g., volumetric muscle loss, cardiotoxin), and diseases (e.g., Duchenne muscular dystrophy) may require context-specific release kinetics of Wnt7a to achieve desired therapeutic effects. Furthermore, using the PEG-4MAL hydrogel platform, physical and biochemical properties, including matrix stiffness, cell-adhesive ligand presentation, structure, and degradability, can be systematically modulated to create a cell-instructive microenvironment $(16,25)$. Here, we demonstrate that the engineered PEG-4MAL hydrogel supports the proliferation, and migration of primary MuSCs in the presence of Wnt7a (Fig. 5). Local co-delivery of MuSCs and Wnt7a using the engineered PEG-4MAL hydrogel to the injured muscle is also feasible (Fig. 6). In subsequent studies, it would be important to evaluate the efficacy of this strategy in other clinically-relevant models of muscle injuries and diseases.

Wnt7a induces myofiber hypertrophy through the Akt/mTOR pathway (8) and symmetric expansion of MuSCs through the planar cell polarity pathway $(6,10)$. Furthermore, pre-treatment of MuSCs with Wnt7a stimulates cell 
migration and engraftment potential, ultimately leading to significantly increased muscle function (7). In this study, we show that Wnt7a released from the hydrogel retains its bioactivity, promotes myofiber hypertrophy, and increases the number of Pax $7^{+}$MuSCs present at the injury site (Fig. 2 and 4). Co-delivery of MuSCs with Wnt7a using the engineered hydrogel also increased the migration distance from the delivery site, suggesting that Wnt7a also increases the MuSC dispersion during the engraftment process when delivered using the hydrogel (Fig. 6c and e). In addition, although previous work has demonstrated that Wnt7a treatment significantly increases the engraftment potential of MuSCs (7), co-delivery of MuSCs with Wnt7a had an insignificant effect on the number of GFP fibers engrafted (Fig. 6c and d). This suggests that the differences in the injury model (e.g., cardiotoxin vs. freeze injury), mode of delivery (e.g., direct injection vs. supra-muscular casting), and use of cell delivery vehicle may influence how the cells engraft into the host muscle upon transplantation.

In summary, we engineered a synthetic PEG-4MAL hydrogel that facilitates the co-delivery of Wnt7a and MuSCs for accelerated skeletal muscle healing in conditions where direct intramuscular injection may be challenging. The maleimide-thiol interaction enables adhesion of Wnt7a and MuSC-encapsulated hydrogel to the freeze-injured muscle surface, and the hydrogel-encapsulated Wnt7a significantly increases muscle fiber hypertrophy, endogenous MuSC expansion, and exogenous MuSC migration during the engraftment process. This work establishes a proof-of-concept biomaterial-mediated therapeutic strategy for promoting skeletal muscle regeneration in the context of traumatic muscle injuries and neuromuscular diseases. 


\section{ACKNOWLEDGMENTS}

We thank the Physiological Research Laboratory and core facilities at the Parker H. Petit Institute for Bioengineering and Bioscience at the Georgia Institute of Technology for the use of shared equipment and services. Research reported in this publication was supported by the National Institutes of Health under Award Numbers F32HL140821, R01AR062368, and R21AR072287. The content is solely the responsibility of the authors and does not necessarily represent the official views of the National Institutes of Health. The authors declare no competing interests.

\section{AUTHOR CONTRIBUTIONS}

W. M. Han, A. J. García, and Y. C. Jang designed the study. W. M. Han, M. Mohiuddin, and S. E. Anderson performed the experiments. W. M. Han, A. J. García, and Y. C. Jang wrote the manuscript. All authors reviewed the manuscript. 


\section{REFERENCES}

1. Corona, B. T., Wenke, J. C., and Ward, C. L. (2016) Pathophysiology of Volumetric Muscle Loss Injury. Cells Tissues Organs (Print) 202, 180-188

2. Feige, P., Brun, C. E., Ritso, M., and Rudnicki, M. A. (2018) Orienting Muscle Stem Cells for Regeneration in Homeostasis, Aging, and Disease. Cell Stem Cell 23, 653-664

3. Corona, B. T., Rivera, J. C., Owens, J. G., Wenke, J. C., and Rathbone, C. R. (2015) Volumetric muscle loss leads to permanent disability following extremity trauma. J Rehabil Res Dev 52, 785-792

4. Garg, K., Ward, C. L., Hurtgen, B. J., Wilken, J. M., Stinner, D. J., Wenke, J. C., Owens, J. G., and Corona, B. T. (2015) Volumetric muscle loss: persistent functional deficits beyond frank loss of tissue. J. Orthop. Res. 33, 40-46

5. Oliva, F., Via, A. G., Kiritsi, O., Foti, C., and Maffulli, N. (2014) Surgical repair of muscle laceration: biomechanical properties at 6 years follow-up. Muscles Ligaments Tendons J 3, 313-317

6. Bentzinger, C. F., Wang, Y. X., von Maltzahn, J., Soleimani, V. D., Yin, H., and Rudnicki, M. A. (2013) Fibronectin regulates Wnt7a signaling and satellite cell expansion. Cell Stem Cell 12, 75-87

7. Bentzinger, C. F., von Maltzahn, J., Dumont, N. A., Stark, D. A., Wang, Y. X., Nhan, K., Frenette, J., Cornelison, D. D. W., and Rudnicki, M. A. (2014) Wnt7a stimulates myogenic stem cell motility and engraftment resulting in improved muscle strength. J. Cell Biol. 205, 97-111

8. von Maltzahn, J., Bentzinger, C. F., and Rudnicki, M. A. (2011) Wnt7a-Fzd7 signalling directly activates the Akt/mTOR anabolic growth pathway in skeletal muscle. Nat. Cell Biol. 14, 186-191

9. von Maltzahn, J., Renaud, J.-M., Parise, G., and Rudnicki, M. A. (2012) Wnt7a treatment ameliorates muscular dystrophy. Proc. Natl. Acad. Sci. U.S.A. 109, 20614-20619

10. Le Grand, F., Jones, A. E., Seale, V., Scimè, A., and Rudnicki, M. A. (2009) Wnt7a activates the planar cell polarity pathway to drive the symmetric expansion of satellite stem cells. Cell Stem Cell 4, 535-547

11. Lin, C.-H., Lin, Y.-T., Yeh, J.-T., and Chen, C.-T. (2007) Free Functioning Muscle Transfer for Lower Extremity Posttraumatic Composite Structure and Functional Defect. Plastic and Reconstructive Surgery 119, 2118-2126

12. Wechselberger, G., Ninkovic, M., Pülzl, P., and Schoeller, T. (2006) Free functional rectus femoris muscle transfer for restoration of knee extension and defect coverage after trauma. Journal of Plastic, Reconstructive \& Aesthetic Surgery 59, 994-998

13. Li, J. and Mooney, D. J. (2016) Designing hydrogels for controlled drug delivery. Nat Rev Mater 1

14. Han, W. M., Jang, Y. C., and García, A. J. (2017) Engineered matrices for skeletal muscle satellite cell engraftment and function. Matrix Biol. 60-61, 96-109

15. Mitrousis, N., Fokina, A., and Shoichet, M. S. (2018) Biomaterials for cell transplantation. Nature Reviews Materials 3, 441

16. Han, W. M., Anderson, S. E., Mohiuddin, M., Barros, D., Nakhai, S. A., Shin, E., Amaral, I. F., Pêgo, A. P., García, A. J., and Jang, Y. C. (2018) Synthetic matrix enhances transplanted satellite cell engraftment in dystrophic and aged skeletal muscle with comorbid trauma. Sci Adv 4, eaar4008

17. García, A. J. (2014) PEG-maleimide hydrogels for protein and cell delivery in regenerative medicine. Ann Biomed Eng 42, 312-322 
18. Phelps, E. A., Enemchukwu, N. O., Fiore, V. F., Sy, J. C., Murthy, N., Sulchek, T. A., Barker, T. H., and García, A. J. (2012) Maleimide cross-linked bioactive PEG hydrogel exhibits improved reaction kinetics and cross-linking for cell encapsulation and in situ delivery. Adv. Mater. Weinheim 24, 64-70, 2

19. Schindelin, J., Arganda-Carreras, I., Frise, E., Kaynig, V., Longair, M., Pietzsch, T., Preibisch, S., Rueden, C., Saalfeld, S., Schmid, B., Tinevez, J.-Y., White, D. J., Hartenstein, V., Eliceiri, K., Tomancak, P., and Cardona, A. (2012) Fiji: an open-source platform for biological-image analysis. Nat. Methods 9, 676-682

20. Lamprecht, M. R., Sabatini, D. M., and Carpenter, A. E. (2007) CellProfiler: free, versatile software for automated biological image analysis. BioTechniques 42, 71-75

21. Phelps, E. A., Headen, D. M., Taylor, W. R., Thulé, P. M., and García, A. J. (2013) Vasculogenic bio-synthetic hydrogel for enhancement of pancreatic islet engraftment and function in type 1 diabetes. Biomaterials 34, 4602-4611

22. Weaver, J. D., Headen, D. M., Hunckler, M. D., Coronel, M. M., Stabler, C. L., and García, A. J. (2018) Design of a vascularized synthetic poly(ethylene glycol) macroencapsulation device for islet transplantation. Biomaterials 172, 5465

23. Weaver, J. D., Headen, D. M., Aquart, J., Johnson, C. T., Shea, L. D., Shirwan, H., and García, A. J. (2017) Vasculogenic hydrogel enhances islet survival, engraftment, and function in leading extrahepatic sites. Sci Adv 3, e1700184

24. Shekaran, A., García, J. R., Clark, A. Y., Kavanaugh, T. E., Lin, A. S., Guldberg, R. E., and García, A. J. (2014) Bone regeneration using an alpha 2 beta 1 integrin-specific hydrogel as a BMP-2 delivery vehicle. Biomaterials 35, 5453-5461

25. Cruz-Acuña, R., Quirós, M., Farkas, A. E., Dedhia, P. H., Huang, S., Siuda, D., García-Hernández, V., Miller, A. J., Spence, J. R., Nusrat, A., and García, A. J. (2017) Synthetic hydrogels for human intestinal organoid generation and colonic wound repair. Nat. Cell Biol. 19, 1326-1335 
bioRxiv preprint doi: https://doi.org/10.1101/569921; this version posted March 6, 2019. The copyright holder for this preprint (which was not certified by peer review) is the author/funder, who has granted bioRxiv a license to display the preprint in perpetuity. It is made available under aCC-BY-NC-ND 4.0 International license.

\section{a Wnt7a-releasing hydrogel}

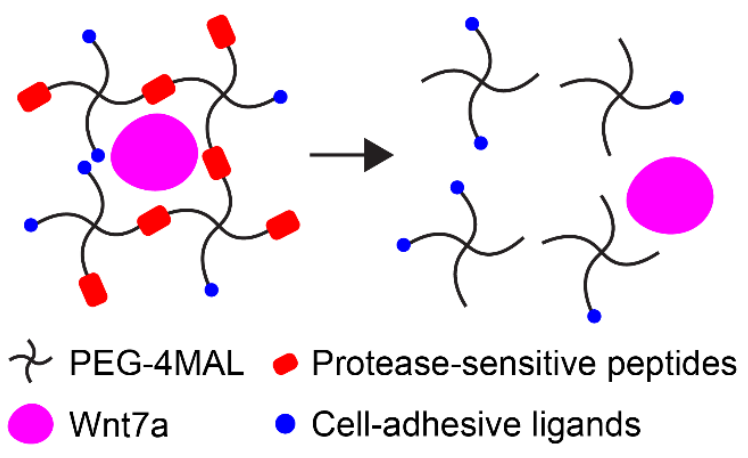

- 0 - 4\% PEG-4MAL + Collagenase

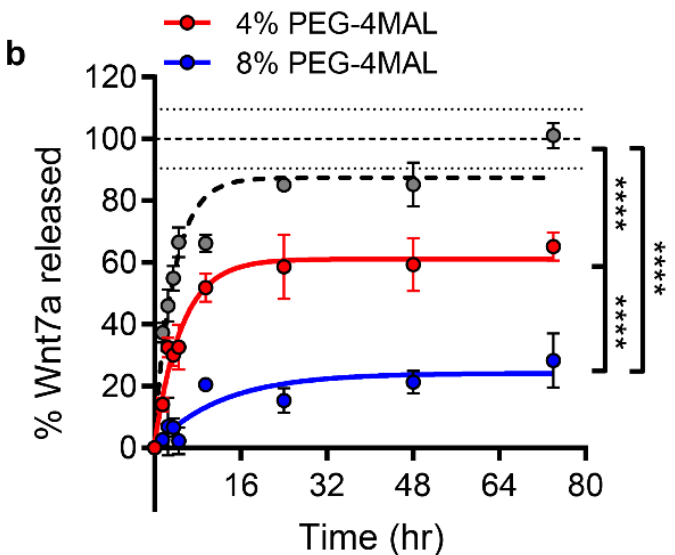

Figure 1. Synthetic PEG-4MAL hydrogel for controlled release of Wnt7a. (a) Schematic diagram of Wnt7a-releasing PEG-4MAL hydrogel. (b) Passive and proteolytic Wnt7a release profile with one-phase association fit. Extra sum of squares F-test for comparing curve fit. Mean \pm SEM. $n=4$ hydrogels/condition. ${ }^{* * * *} p<0.0001$. 
bioRxiv preprint doi: https://doi.org/10.1101/569921; this version posted March 6, 2019. The copyright holder for this preprint (which was not certified by peer review) is the author/funder, who has granted bioRxiv a license to display the preprint in perpetuity. It is made available under aCC-BY-NC-ND 4.0 International license.
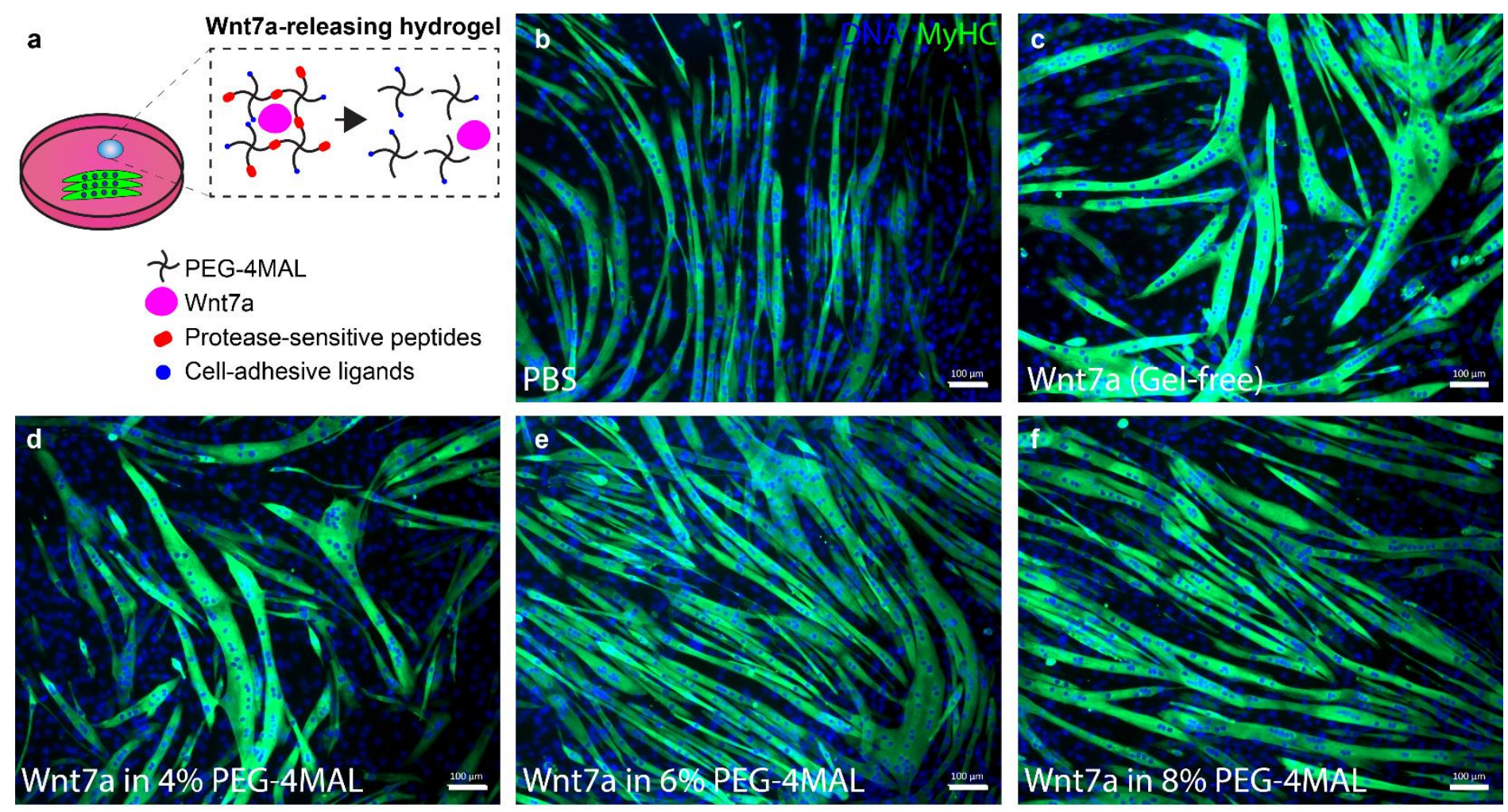

g

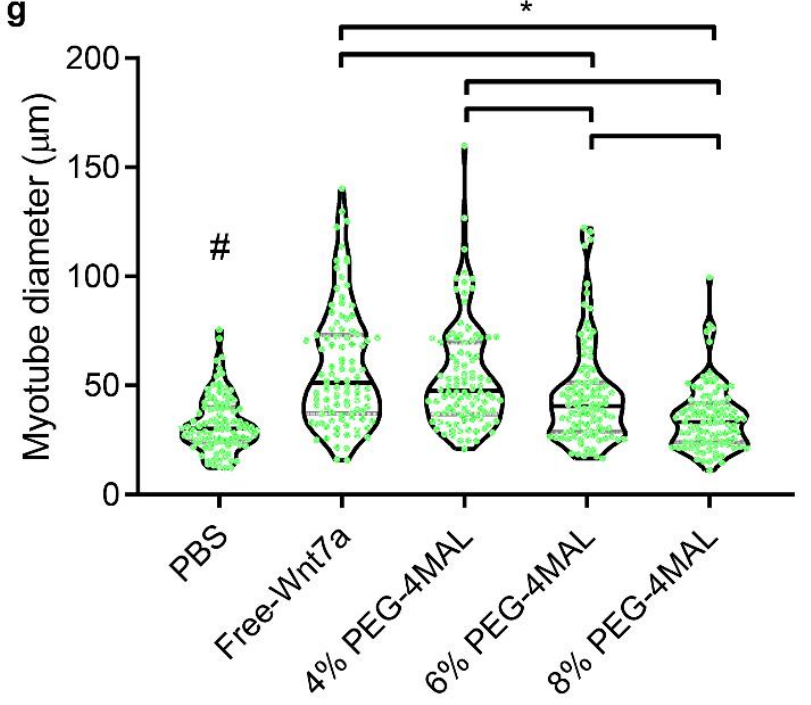

h

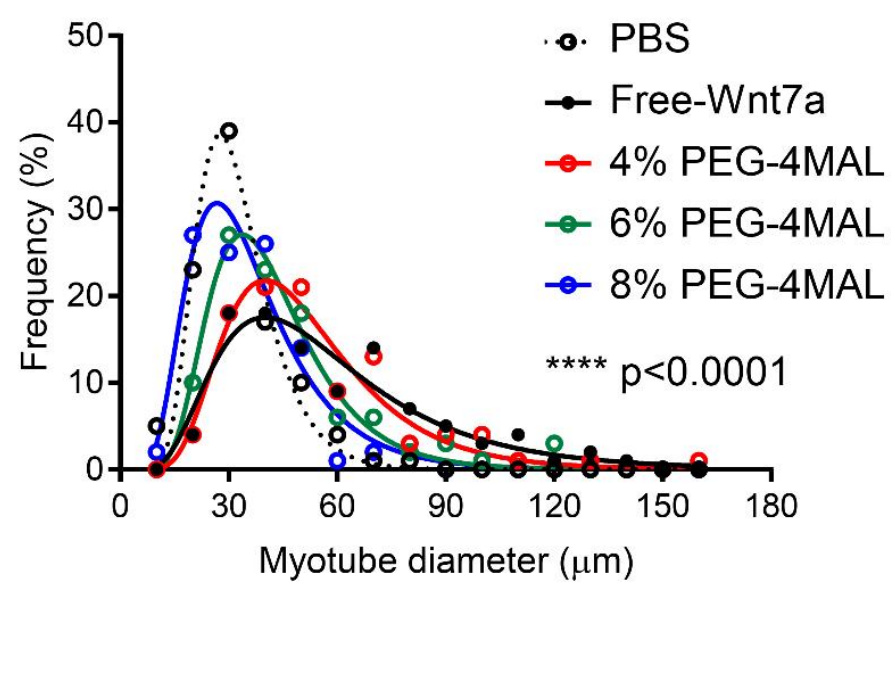

Figure 2. Hydrogel-released Wnt7a retains its bioactivity in vitro. (a) Schematic diagram of the experiment.

Differentiating C2C12 myotubes treated with (b) PBS, (c) Wnt7a (gel-free), (d) Wnt7a in 4\% PEG-4MAL hydrogel, (e) Wnt7a in 6\% PEG-4MAL hydrogel, and (f) Wnt7a in 8\% PEG-4MAL hydrogel. Day 5. Scale bar $100 \mu \mathrm{m}$. (g) Violin plot of myotube diameter. $\mathrm{n}=100$ myotubes/condition. Kruskal-Wallis test with Dunn's multiple comparisons test. $\# p<0.001$ vs. free-Wnt7a, 4\% PEG-4MAL, and 6\% PEG-4MAL. ${ }^{*} p<0.05$. (h) Histogram of myotube diameter distribution with the logGaussian fit. Extra sum of squares F-test for comparing curve fit. ${ }^{* * * *} p<0.0001$. 
bioRxiv preprint doi: https://doi.org/101101/569921: this version posted March 6, 2019. The copyright holder for this preprint (which was not certified by peer review) is the author/funder, who has granted bioRxiv a license to display the preprint in perpetuity. It is made available under aCC-BY-NC-ND 4.0 International license.

a

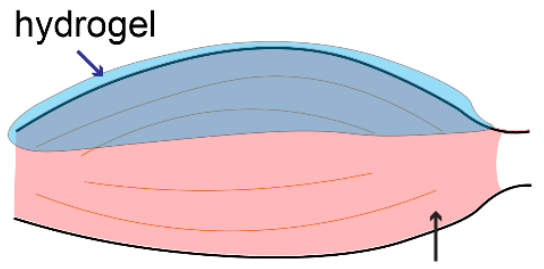

muscle

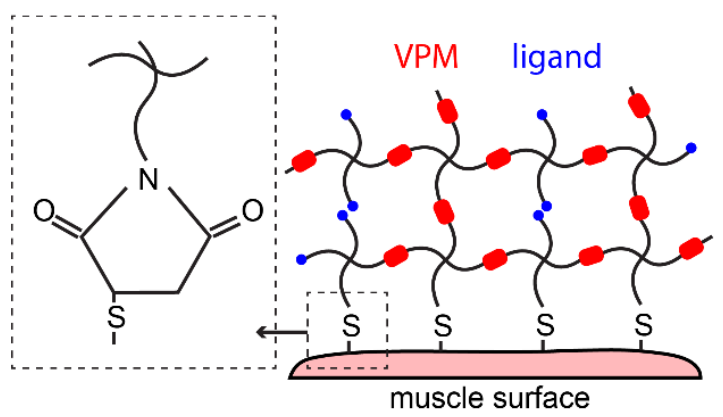

b
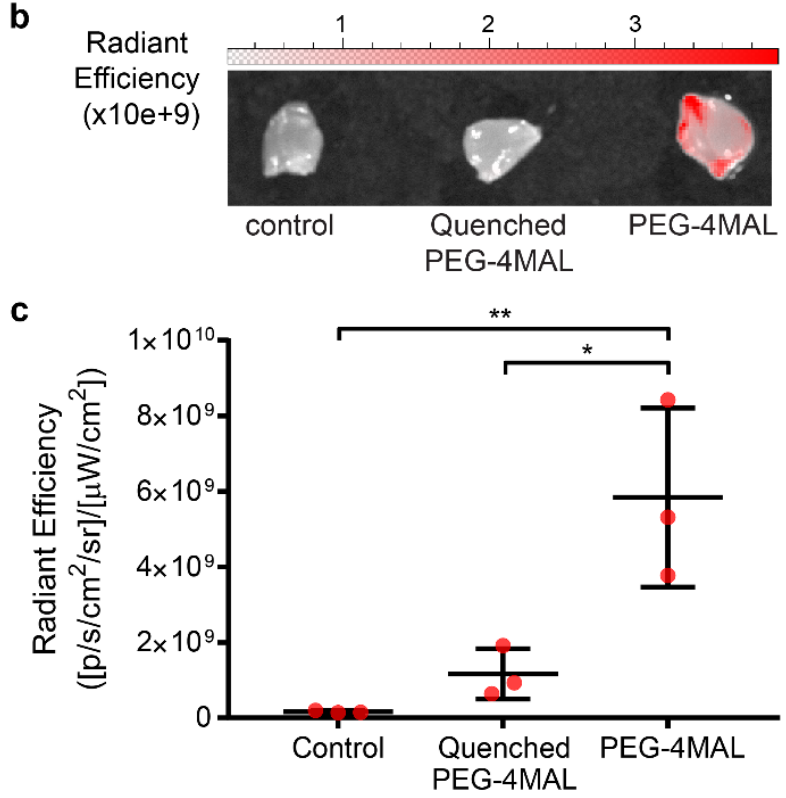

Figure 3. PEG-4MAL macromer adheres to the muscle surface. (a) Schematic diagram illustrating the ability of PEG4MAL hydrogel to adhere to the muscle surface via maleimide-thiol reaction. (b) Representative IVIS images of muscles treated control (saline), quenched PEG-4MAL macromers conjugated with DyLight ${ }^{\mathrm{TM}}$-755-labeled RDG peptides, and PEG-4MAL macromers conjugated with DyLight ${ }^{\mathrm{TM}}$-755-labeled RDG peptides. (c) Quantification of radiant efficiency. One-way ANOVA with Tukey's multiple comparisons test. ${ }^{*} p<0.05,{ }^{* *} p<0.01$. 
bioRxiv preprint doi: https://doi.org/10.1101/569921; this version posted March 6, 2019. The copyright holder for this preprint (which was not certified by peer review) is the author/funder, who has granted bioRxiv a license to display the preprint in perpetuity. It is made available under

a

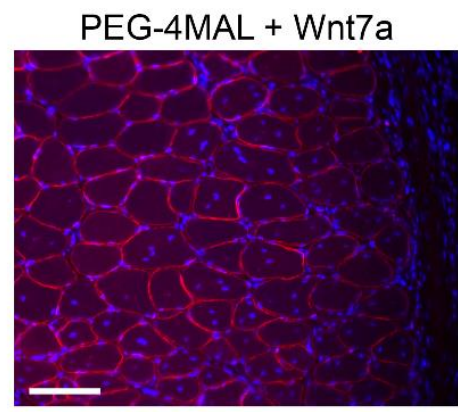

b

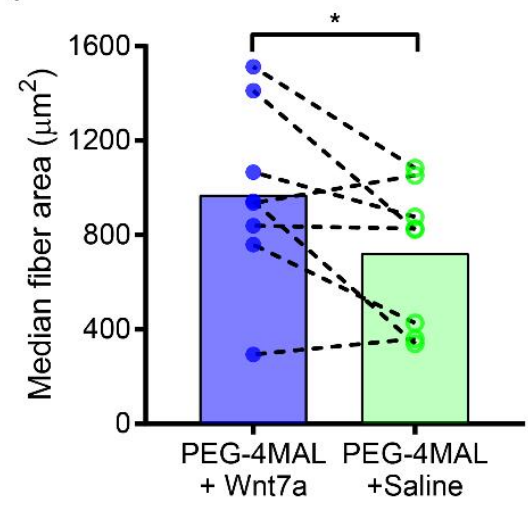
aCC-BY-NC-ND 4.0 International license.

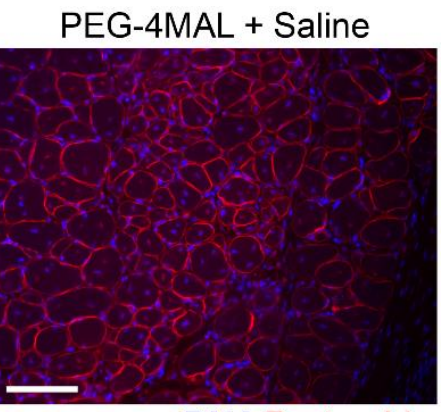

DNA Dystrophin

C

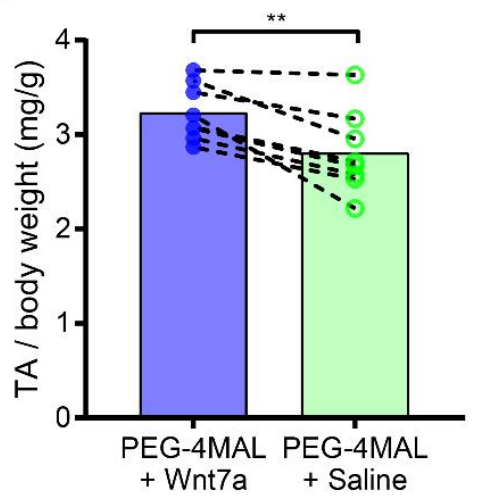

d PEG-4MAL + Wnt7a

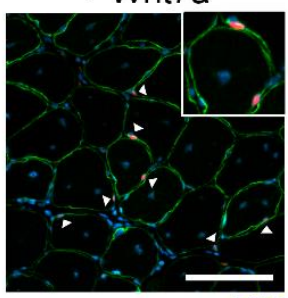

DNA Dystrophin Pax7

e

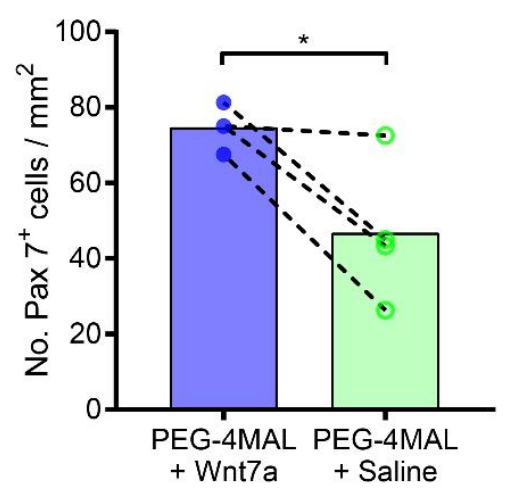

Figure 4. Engineered PEG-4MAL hydrogel enables effective local delivery of Wnt7a to the injured skeletal muscle. (a) Representative images of TA muscles treated with PEG-4MAL hydrogels loaded with Wnt7a or saline. Day 14 posttreatment. Scale bar $100 \mu \mathrm{m}$. (b) Median fiber area. Day 14 post-treatment. Two-tailed paired t-test. (c) Mass of TA muscles normalized to the respective body weight. Day 14 post-treatment. Two-tailed paired t-test. (d) Representative images of TA muscles treated with PEG-4MAL hydrogels loaded with Wnt7a or saline. Arrows indicate Pax ${ }^{+}$muscle stem cells. Day 14 post-treatment. Scale bar $100 \mu \mathrm{m}$. (e) The number of Pax $7^{+}$muscle stem cells per $\mathrm{mm}^{2}$. Day 14 posttreatment. Two-tailed paired t-test. ${ }^{*} p<0.05,{ }^{* *} p<0.01$. 
bioRxiv preprint doi: https://doi.org/10.1101/569921; this version posted March 6, 2019. The copyright holder for this preprint (which was not certified by peer review) is the author/funder, who has granted bioRxiv a license to display the preprint in perpetuity. It is made available under aCC-BY-NC-ND 4.0 International license.
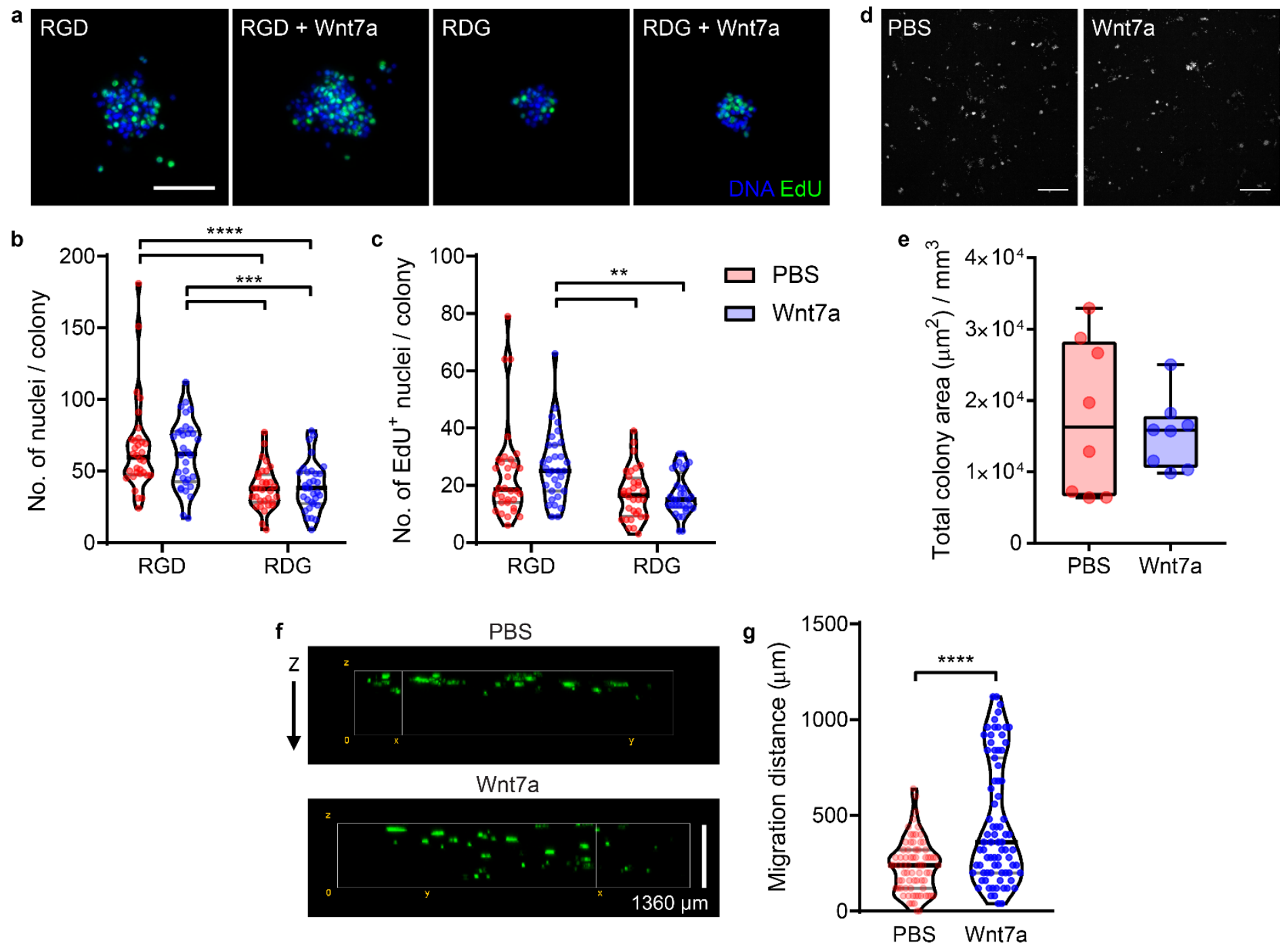

Figure 5. Wnt7a does not increase MuSC proliferation but promotes muscle stem cells migration through the engineered PEG-4MAL hydrogel. (a) Representative confocal images of myogenic colonies formed in RGD-presenting PEG-4MAL hydrogel, RGD-presenting PEG-4MAL hydrogel with Wnt7a, RDG-presenting PEG-4MAL hydrogel, and RDGpresenting PEG-4MAL hydrogel with Wnt7a. Scale bar $50 \mu \mathrm{m}$. (b) Violin plot of myogenic colony size. Two-way ANOVA with Sidak's multiple comparisons test. $n=32$ colonies/condition. (c) Violin plot of EdU quantification. Two-way ANOVA with Sidak's multiple comparisons test. $n=32$ colonies/condition. (d) Representative confocal images of nuclei-stained myogenic colonies formed in RGD-presenting PEG-4MAL hydrogels with or without Wnt7a. Scale bar $500 \mu \mathrm{m}$. (e) Colony density quantified by total colony area per volume. (f) 3D projection of GFP+ muscle stem cells migrating through the hydrogel from the top surface. (g) Violin plot of cellular migration distance. Day 3. n=79-74 cells/condition. MannWhitney test. $* * p<0.01, * * * p<0.001, * * * * p<0.0001$. 
bioRxiv preprint doi: https://doi.org/10.1101/569921; this version posted March 6, 2019. The copyright holder for this preprint (which was not certified by peer review) is the author/funder, who has granted bioRxiv a license to display the preprint in perpetuity. It is made available under

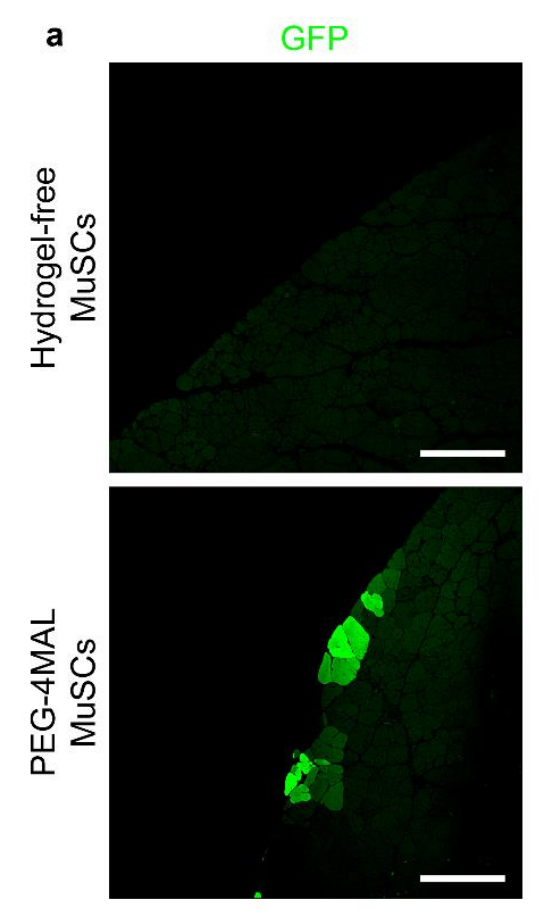
aCC-BY-NC-ND 4.0 International license.
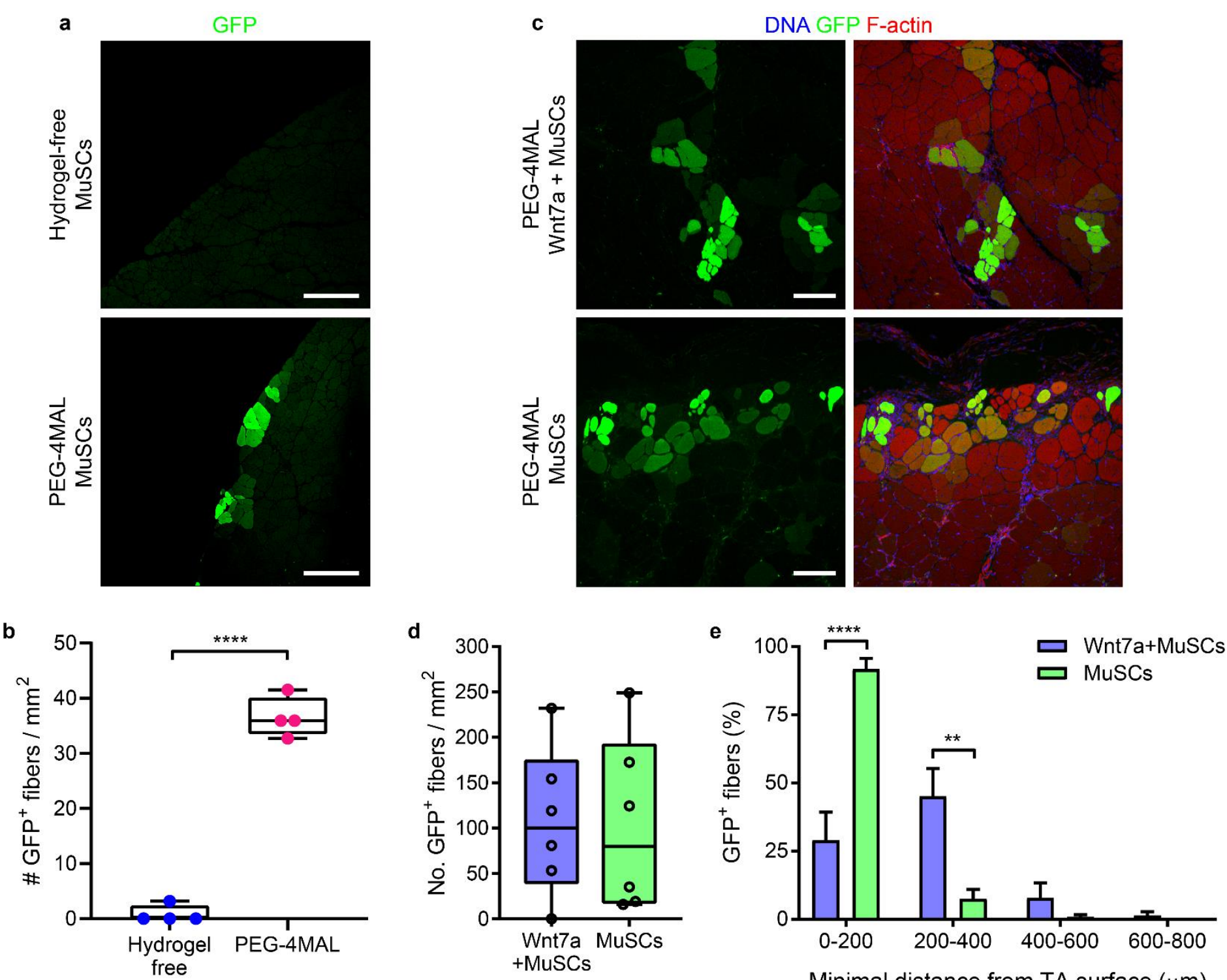

Minimal distance from TA surface $(\mu \mathrm{m})$

Figure 6. Wnt7a increases migration potential of PEG-4MAL-released muscle stem cells in vivo. (a) Representative confocal images of TA muscles treated with muscle stem cells alone and muscle stem cells delivered using the engineered PEG-4MAL hydrogel. 40,000 cells delivered per TA. Scale bar $250 \mu \mathrm{m}$. (b) The number of engrafted GFP ${ }^{+}$ fibers. Two-tailed unpaired t-test. (c) Representative confocal images of TA muscles treated with muscle stem cells encapsulated in PEG-4MAL hydrogels with or without Wnt7a. 50,000 cells delivered per TA. Scale bar $100 \mu \mathrm{m}$. (d) The number of engrafted GFP' fibers. Two-tailed paired t-test. (e) Histogram of GFP fibers (\%) as a function of minimal distance from the TA surface. Two-way repeated measures ANOVA with Sidak's multiple comparisons test. ${ }^{* *} p<0.01$, $* * * * p<0.0001$. 